\title{
ON THE eCAADe SIGraDi JOINT CONFERENCE 2019
}

The 2019 Conference in Porto joins, for the first time, two major Associations in the field of Digital Technologies in Architecture - eCAADe (Europe) and SIGraDi (Iberoamérica) - to debate together about Architecture in the Age of the $4^{\text {th }}$ Industrial Revolution.

\section{Background}

The beginning of this journey started in 2015. Following the organization of the $1^{\text {st }}$ eCAADe Regional International Workshop (RIW) "Future Traditions" at FAUP in 2013, José Pinto Duarte challenged José Pedro Sousa to submit a proposal to host the Annual eCAADe Conference in Porto. Despite the enormous difficulty and responsibility of undertaken such task, that possibility looked very opportune for the Faculty of Architecture of the University of Porto (FAUP) and for the City of Porto. Widely recognized for its pedagogy and the architecture of Álvaro Siza and Souto de Moura, the event could strengthen the relevance of technology in the School and stimulate its singular potential to bridge traditions with digital innovation. On the other hand, aside with its rich heritage legacy, the city of Porto has become a vibrant place for the arts, architecture, culture and entrepreneurship, and such fact sets the perfect atmosphere for hosting the event.

Thus, in 2016, a submission to debate the place of Architecture in the Age of the $4^{\text {th }}$ Industrial Revolution was submitted to the eCAADe Council and, during the $34^{\text {th }}$ eCAADe Conference in Oulu, the Faculty of Architecture of the University of Porto (FAUP) was then elected to organize the $37^{\text {th }}$ eCAADe Conference in 2019 in the city of Porto, following the editions in Rome (2017) and Lodz (2018).

Later, in the other side of the Atlantic Ocean, during the XXI SIGraDi Conference in Concepcion (Chile 2017), Gonçalo Castro Henriques made a proposal to SIGraDi Council CEI that was accepted, to invite the FAUP to also organize the 2019 Conference in the city of Porto. That idea came into life because Portugal, as well as Spain, is officially part of the Iberoamerican 
Community. Following the editions in São Carlos SP (Brazil 2018), and Concepcion (Chile 2017), this was a unique opportunity to bring SIGraDi for the first time to the Iberian Peninsula.

Realizing the overlap of the two Conferences in the city of Porto, the possibility to create a Joint event in Portugal emerged as an exciting opportunity for bringing together the two communities and debate a global theme. Such one-time collaborative initiative between eCAADe and SIGraDi would celebrate the openness and exchange of knowledge between countries across continents in a current moment where frontiers and walls seem to be gaining a renewed impetus.

However, moving forward with such challenge required a series of conversations between eCAADe and SIGraDi Councils to find a common ground regarding both associations' traditions and culture. Over several months, formal and implementations issues were discussed to overcome the differences and make possible the ambitious joint initiative. This was facilitated by the fact that the Chairs from both Associations were from the city of Porto, and have collaborated since long ago. After reaching a formal agreement, the Councils of eCAADe and SIGraDi appointed a joint Commission to assist the organization of the Conference. Birgul Colakoglu, Joachim Kieferle, Henri Achten, Rudi Stouffs (eCAADe) and Fernando Garcia Amen, Marcelo Bernal, Pablo Herrera and Rodrigo Martín Iglésias (SIGraDi) were unbeatable with their support and guidance over the months of preparations.

\section{Developments}

The unique features of the joint event triggered an immense curiosity and interest in the event. As a result, the Call for Papers received $\mathbf{6 4 9}$ extended abstracts submitted from $\mathbf{5 0}$ countries. Such number set a new record in the history of both associations and became a serious challenge for the organization. 


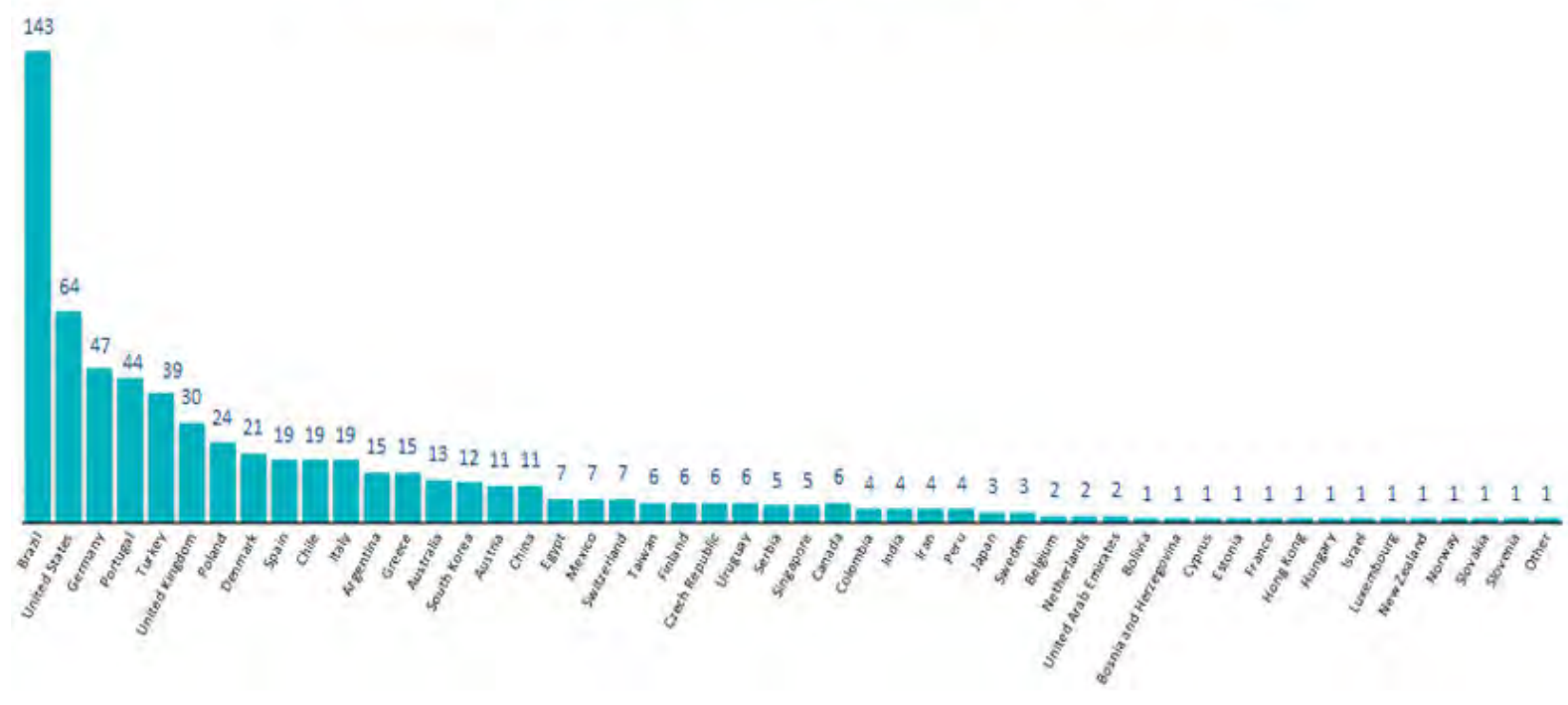

Call for Papers results - submissions / country

The review of the extended abstracts (length of 1000 to 1500 words plus 5-10 references and one image) followed the high-quality control process well established in these Conferences. Each submission had to be strictly anonymous and to avoid any affiliations and was evaluated by three reviewers coming from institutions other than the authors. Thanks to eCAADe, we were able to use the OpenConf System to carefully manage the entire anonymous submission and evaluation process.

Reviewing such large number of abstracts was only possible with the professionalism and generosity of our Scientific Committee composed by 203 reviewers from $\mathbf{4 0}$ countries. To accomplish the 1947 required reviews, we created a special group of experienced reviewers the Expert Group / Champion reviewers - who accepted to evaluate a greater number of submissions than usual, and to whom we are especially thankful. We pay tribute to each of the reviewers in the reviewers list.

The review process was concluded with the selection of $\mathbf{2 6 9}$ abstracts from $\mathbf{3 9}$ countries for further development as a Full Paper. The submission process of the Full Papers was then handled through the Proceedings Platform and resulted in the final publication of 221 works. 
In parallel to the Conference preparations, we organized a Call for Workshops that received 21 proposals. Out of these, 6 workshops were selected and added to the 3 Workshops proposed by our main sponsors Autodesk, Graphisoft and Dassault Systèmes. Following the tradition of eCAADe and SIGraDi Conferences, and the spirit of the current edition, a Joint PhD Workshop was organized by Wolfgang Dokonal (eCAADe) and Gabriela Celani (SIGraDi). The PhD Workshop Committee selected a total of $10 \mathrm{PhD}$ students to come to Porto and discuss their research with an experienced team of international critics. The 9 Workshops and the PhD Workshop were featured as Pre-Conference events happening from 9-10 September.

\section{Conference}

The eCAADe SIGraDi Joint Conference attracted 300 participants to the Faculty of Architecture of the University of Porto (FAUP) from 11-13 September. For organizing the sessions, we defined 6 main themes to frame the different specific session topics. Following this organization, 221 presentations unfolded through 4 parallel sessions over the three conference days.

\begin{tabular}{ll}
\hline Design & Algorithmic and Parametric \\
& Artificial Intelligence \\
& Collaboration and Participation \\
& Generative Systems \\
& Shape Grammars and Ruled Based Systems \\
\hline Matter & Additive Manufacturing \\
& Digital Production and Robotics \\
& Fabrication and Construction \\
& Material Studies and Innovation \\
\hline Data & Building Information Modelling \\
& City Information Modelling and GIS \\
& Cultural Heritage \\
& Smart Cities \\
\hline Interaction & Human-Computer Interaction \\
& Responsive environments \\
\hline Simulation & Prediction and Evaluation \\
& Virtual and Augmented Reality \\
\hline Challenges & Art and Digital Poesies \\
& Big Data and Analytics \\
& Education and Research \\
& History and Future CAAD \\
& Sustainability +Cultural Heritage \\
\hline
\end{tabular}

Theme structure ruling the Conference Session topics 
Such structure is also reflected in the organization of the current publication, where each volume is matching each Conference day.

The four Keynotes were invited to provide particular, but complementary, visions addressing the spirit and theme of the event. Thus, we were grateful to count with:

- Professor Mario Carpo (UCL), discussing the theory and critic of the digital revolution;

- BIG (Bjarke Ingles Group) Associate and BCN Design Director João Albuquerque, featuring the work of one of the most distinguished international practices today;

- Professor Gabriela Celani (UNICAMP) tracing the panorama of the 4th Industrial revolution in the Iberoamérica;

- Architect Marta Campos (Porto-based office), revealing the fusion between tradition and digital innovation on a young, small and local practice.

In order to establish a special moment for collective discussion, Professor José Pinto Duarte (PSU) was invited to Chair of a Roundtable about the Architecture in the Age of the $4^{\text {th }}$ Industrial Revolution. To host all these special moments with a wider audience, we resort the Theatre Campo Alegre, nearby FAUP.

Aside with the Conference sessions, a series of special social programs were also organized for each night, flowing from the City Hall of Porto, the Fenianos Club, the Douro River, the Alfândega Building, the Nuno Centeno Art Gallery, and the City Downtown.

We this intensive and vast program, we tried to stimulate the scientific exchange between peers from each Association, but also the establishment of friendship links between them. Although from next year on each association will return to their independent annual Conferences, we hope this joint initiative can trigger new productive collaborations between the two communities and thus extend the spirit of the eCAADe SIGraDi 2019 over time.

José Pedro Sousa, Gonçalo Castro Henriques and João Pedro Xavier eCAADe SIGraDi 2019 Conference Chairs 